\title{
基于吡咯并吡咯二酮核心的菲二酰亚胺类受体分子的合成及光伏性能
}

\author{
胡瑜辉 $a$ 武文林 ${ }^{b}$ 于立扬*, $b$ 骆开均 $*, a$ \\ 徐小鹏 $b$ 李瑛 ${ }^{b}$ 彭强 $*, b$ \\ $\left({ }^{a}\right.$ 四川师范大学化学与材料科学学院 成都 610066$)$
}

$\left({ }^{b}\right.$ 四川大学化学学院 绿色化学与技术教育部重点实验室 高分子材料工程国家重点实验室＼cjkstart成都 610064)

\begin{abstract}
摘要 荘二酰亚胺类小分子由于其固有的强分子聚集特性, 导致活性层形貌难于调控, 器件效率相对于近年来报道的 受体-给体-受体型稠环小分子受体一直处于劣势. 针对这一关键问题, 我们设计并合成了三个以吡咯并吡咯二酮为中 心核的双臂型和四臂型芢二酰亚胺类小分子受体. 其中, c-PDI2 和 nc-PDI2 两个双臂型分子分别将两个芢二酰亚胺臂 置于吡咯并吡咯二酮核心骨架的碳取代位和氮取代位; 四臂型 PDI4 是将四个芢二酰亚胺臂置于吡咯并吡咯二酮核心 骨架的四个取代位. 通过对三个受体小分子的光谱吸收、能级水平、薄膜形貌以及光伏性能的详细研究, 发现三个受 体小分子都拥有扭曲的分子结构并由此带来无定形薄膜形貌，表明其分子聚集趋势得到了有效的抑制. 相对于双臂型 受体分子, 四臂型 PDI4 具有更强的光吸收能力和电子传输性能, 从而获得了 $8.45 \%$ 的最高光电转换效率, 是 $\mathbf{c - P D I 2}$ 器 件效率的 2 倍和 nc-PDI2 器件效率的 1.5 倍.
\end{abstract}

关键词＼cjkstart聚合物太阳能电池；非富勒烯受体；菲二酰亚胺；三维构型；高效率

\section{Synthesis and Photovoltaic Properties of Perylene Diimide Based Small Molecular Acceptors with a Diketopyrrolopyrrole Core}

\author{
$\mathrm{Hu}$, Yuhui $^{a} \quad \mathrm{Wu}$, Wenlin $^{b} \quad$ Yu, Liyang*,b \\ Luo, Kaijun*,a Xu, Xiaopeng ${ }^{b}$ Li, Ying ${ }^{b}$ Peng, Qiang* ${ }^{*, b}$ \\ $\left({ }^{a}\right.$ College of Chemistry and Materials Science, Sichuan Normal University, Chengdu 610066, China) \\ ( ${ }^{b}$ Key Laboratory of Green Chemistry and Technology of Ministry of Education, College of Chemistry and \\ State Key Laboratory of Polymer Materials Engineering, Sichuan University, Chengdu 610064, China)
}

\begin{abstract}
Polymer solar cells (PSCs) experienced a leap forward recently due to the development of non-fullerene acceptors. These novel acceptor materials possess improved photon absorption ability as well as readily tunable band structures compared to the conventional fullerenes. Perylene diimide (PDI) derivatives were among the first investigated non-fullerene acceptors for PSCs. PDI is widely adopted as building blocks for acceptor materials for its high photon absorption and electron transporting abilities, suitable and tunable energy levels, ease of synthesis and excellent photon stability. However, PDI derivatives are well known for their aggregation tendency to result in poor blend morphology, which leads to lower device efficiency than the acceptor-donor-acceptor type fused ring small molecular acceptors. To address this issue, we designed and synthesized three PDI based small molecular acceptors with a diketopyrrolopyrrole (DPP) core. The c-PDI2 and nc-PDI2 were two-PDI-armed molecules with PDI substituents attached on the carbon or nitrogen atoms of the DPP skeleton, respectively, while PDI4 was a four-PDI-armed molecule. The PDI units were predicted by molecular simulations to be positioned in altered planes forming the twisted $3 \mathrm{D}$ structures, which would reduce the intermolecular aggregation. Based on optical absorption, energy level, blend morphology and photovoltaic performance studies, all three molecules were found with amorphous morphology, which indicated that the aggregation tendency was efficiently suppressed. Among the three molecules, the four-armed PDI4 displayed the flatter structure with broadened electron delocalization which led to significantly increased extinction coefficient and electron transport mobility. The faster electron transport of PDI4 assured the balanced charge transport which yielded into a higher field factor (FF) over $65 \%$ in contrast to the two-armed molecules with FFs under 55\% in the respective PSC devices. With additional aids from the increased photon absorption, PSCs containing PDI4 also generated substantially higher photocurrent. These improvements afforded the highest power conversion efficiency (PCE) as high as $8.45 \%$ among the three PDI derivatives, which was twice and 1.5 times of those of c-PDI2 and nc-PDI2, respectively. In comparison between the two two-armed PDI derivatives, the nitrogen-position-substituted nc-PDI2 delivered higher device performances than the carbon-position-substituted c-PDI2, also thanked to its flatter molecular arrangement
\end{abstract}

*E-mail: liyangyu@scu.edu.cn; luo-k-j007@163.com; qiangpeng@scu.edu.cn

Received July 1, 2020; published August 12, 2020.

Supporting information for this article is available free of charge via the Internet at http://sioc-journal.cn.

Project supported by National Natural Science Foundation of China (Nos. 21825502, 21905185), the Foundation of State Key Laboratory of Polymer Materials Engineering (No. SKLPME 2017-2-04) and the Fundamental Research Funds for the Central Universities (No. YJ201957).

国家自然科学基金(Nos. 21825502，21905185), 高分子材料工程国家重点实验室基金(No. SKLPME 2017-2-04)和中央高校基本科研基金(No. YJ201957)共同项目。 
and broader intra- and intermolecular electron delocalization. In our study, we successfully prevented aggregation of PDI derivatives by constructing 3D molecular structures with multiple PDI units. The numbers and substituting positions of PDIs on the DPP core were also investigated in detail, which provided valuable insights for designing of high performance PDI derivatives for PSCs.

Keywords Polymer solar cells; non-fullerene acceptor; perylene diimide; three-dimensional; structure; high efficiency

\section{1 引言}

聚合物太阳能电池具有可溶液加工、柔性和低成本 等传统太阳能电池无法比拟的优势，是第三代太阳能电 池的典型代表 ${ }^{[1]}$. 近年来, 基于非富勒烯受体的聚合物 太阳能电池突破了传统富勒烯受体相对较弱的光吸收 能力所带来的瓶颈, 极大提高了聚合物太阳能电池的光 电转换效率 $(\mathrm{PCE})^{[2-5]}$. 目前非富勒烯聚合物太阳能电 池的 PCE 已经突破了 $16 \%$, 显示出了其很好的应用前 景 ${ }^{[6-11]}$.

菲二酰亚胺类分子由于具有强的光吸收能力、高的 电子迁移率、适合且可调控的电子能级、易合成和良好 的光化学稳定等优点, 是最早被关注的非富勒烯受体小 分子之一 ${ }^{[2,12-15]}$. 然而菲二酰亚胺类受体小分子也具有 很强的自聚集特性, 所以在溶液加工过程中, 活性层中 受体小分子和给体聚合物之间的相分离尺度远大于数 十纳米的最优范围, 导致严重的载流子复合, 大大限制 了器件效率的进一步提升 ${ }^{[16,17]}$.

已有的研究表明, 将多个菲二酰亚胺单元通过分子 设计连接成非平面结构, 可以有效降低菲二酰亚胺类受 体分子的平面性并抑制由此带来的自聚集倾向. 相对于 首尾相连结构 ${ }^{[18-20]}$, 将连接点放置于菲二酰亚胺的湾位 是比较好的分子结构设计 ${ }^{[21-24]}$. 除了湾位连接可以降低 菲二酰亚胺单元的平面性外, 三维结构的菲二酰亚胺受 体小分子的设计则更有利于抑制其分子自聚集性 ${ }^{[25]}$. 基于这些设计策略，大量的含有两个 ${ }^{[21-24]}$ 、三个 ${ }^{[12,26,27]}$ 、

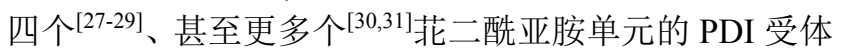
小分子被报道并成功应用于聚合物太阳能电池. 通过对 侯剑辉课题组 2016 年报道的 PPDI ${ }^{[20]}$ 和 H-tri-PDI ${ }^{[19]}$ 、黄 飞课题组 2017 年报道的 P2N2 和 P4N4 $4^{[32]}$ 、Kilwon 课题 组 2018 年报道的 SF-iPDI2 和 SF-iPDI4 $4^{[33]}$ 等 PDI 分子体 系的研究对比中可以发现, 增加分子中菲二酰亚胺单元 的数量, 可以更加有效地提高这类材料的光谱吸收能 力, 进而提升器件的 PCE.

另一方面, 吡咯并吡咯二酮 (DPP) 也具有其很好 的化学稳定性、强的光吸收能力、容易合成和修饰等优 点, 被用于构筑给体-受体 (D-A) 型聚合物材料, 这些 材料已被广泛应用于聚合物太阳能电池 ${ }^{[34,35]}$ 和聚合物 场效应晶体管 ${ }^{[34,36]}$. DPP 也已被应用并构造出多种非富 勒烯受体小分子, 获得了不错的 $\mathrm{PCE}^{[34,37,38]}$. 但是基于 DPP 为核心的菲二酰亚胺类受体小分子目前还没有文 献报道. 目前已报道的大多数菲二酰亚胺衍生物多以给 体单元为中心核 ${ }^{[21,22,27-29]}$, 而以受体单元作为中心核的
菲二酰亚胺衍生物非常少 ${ }^{[24]}$. 由于缺电子的受体骨架 为中心核可以进一步提升受体小分子的电子传输性能, 因此本工作设计制备以 DPP 为中心核的芢二酰亚胺受 体小分子并应用于聚合物有机太阳能电池具有十分重 要的意义.

为此, 我们充分利用 DPP 多个活性连接位点的特 点, 通过苯环 $\pi$ 桥与两个或四个菲二酰亚胺结构单元键 接, 设计合成了 $A_{1}-\pi-A_{2}-\pi-A_{1}$ 型的芢二酰亚胺受体小分 子 c-PDI2, nc-PDI2 和 PDI4, 其分子结构如图 1 所示, 具体的合成路线如图式 1 所示. 其中, c-PDI2 和 nc-PDI2 分别将两个花二酰亚胺结构单元键接在 DPP 的碳取代 位和氮取代位上, 而 PDI4 则是将四个菲二酰亚胺结构 单元键接在 DPP 的四个取代位上. 通过对三个 PDI 受体 小分子的对比, 不仅可以研究 PDI 单元数量对相关性能 的影响, 也可以深入探讨在 DPP 不同位置引入 PDI 单元 对于分子光电性能的影响规律. 研究发现, 四臂型 PDI4 比双臂型 PDI 受体小分子的摩尔消光系数高出一倍以 上, 这极大地提高了分子的吸光能力, 从而为获得器件 更高的短路电流密度 $\left(J_{\mathrm{sc}}\right)$ 奠定了基础. 同时, PDI4 与 窄带隙给体聚合物 PTB7-Th 共混薄膜的电子迁移率也 远大于另外两个基于双臂型 PDI 受体小分子的共混薄 膜, 并且空穴/电子迁移率也更加平衡, 这样降低了器件 中的载流子复合，从而获得了更高的填充因子 (FF). 基 于这些原因, 基于 PDI4 的聚合物太阳能器件获得了最 高的 PCE, 达到 $8.45 \%$, 分别是 c-PDI2 和 nc-PDI2 器件 效率的 2 倍和 1.5 倍. 对比研究两个双臂受体小分子, 在 氮原子上连接 PDI 结构单元的 nc-PDI2 表现出了更强的 电子传输性能, 同时提高了器件的 $J_{\mathrm{sc}}$ 和 $\mathrm{FF}$, 从而表现 出了更高的器件性能.

\section{2 结果与讨论}

\section{1 受体小分子的热性能}

通过热重分析(TGA) 对受体小分子的热稳定性进 行研究 (如图 S1a 所示), c-PDI2, nc-PDI2 和 PDI4 的热 分解温度 (5\%热失重) 分别为 348,349 和 $409{ }^{\circ} \mathrm{C}$, 这表 明了三个受体小分子都具有很好的热稳定性. 在 $50{ }^{\circ} \mathrm{C}$ 到 $250{ }^{\circ} \mathrm{C}$ 的温度区间内, 通过差示扫描量热法(DSC, 图 S1b) 对三个受体小分子的表征, 没有检测到与相转 变和玻璃化温度等相关信息.

\section{2 受体小分子的光物理性能}

三个受体小分子在氯仿溶液中的紫外-可见光 (UV-vis) 吸收光谱见图 2a. c-PDI2、nc-PDI2 和 PDI4 
(a)

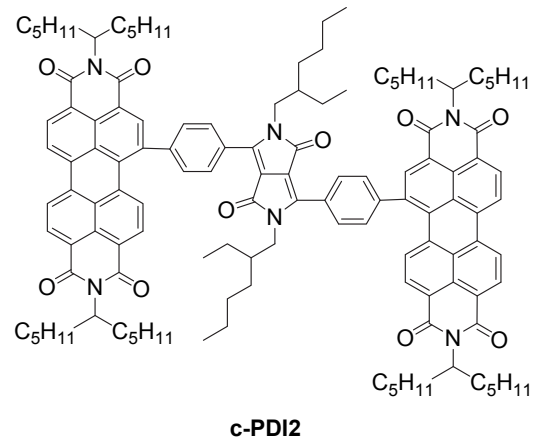

C-PDI2

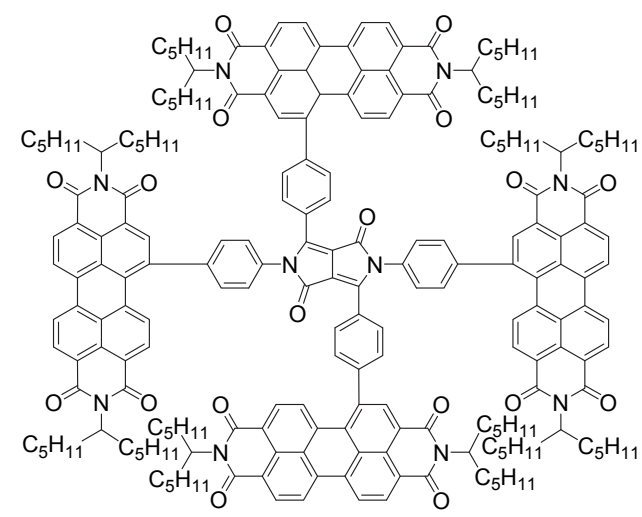

PDI4

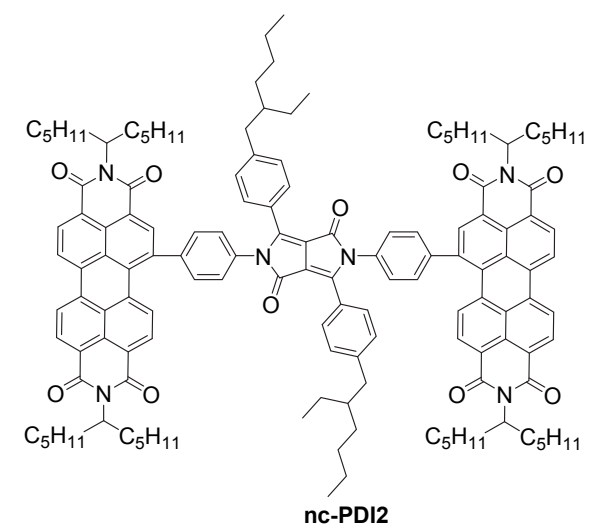

(b)

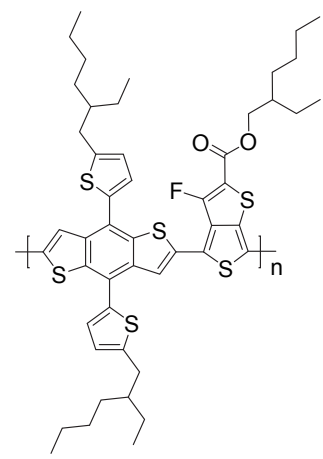

PTB7-Th

图 1 受体小分子 c-PDI2, nc-PDI2, PDI4 和给体聚合物 PTB7-Th 的分子结构

Figure 1 Molecular structures of acceptor molecules c-PDI2, nc-PDI2, PDI4 and donor polymer PTB7-Th

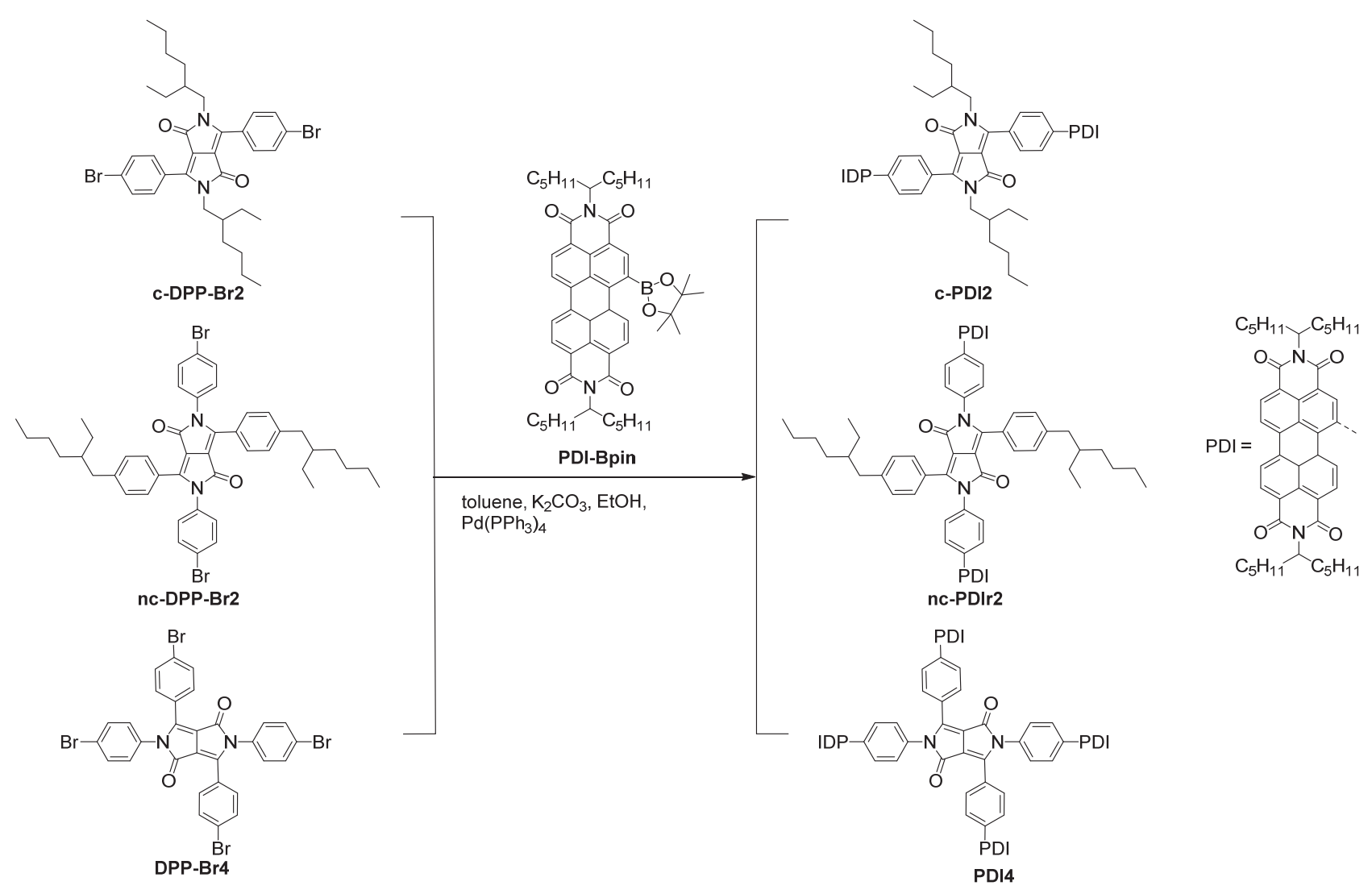

图式 1 受体小分子 c-PDI2, nc-PDI2, PDI4 的合成路线

Scheme 1 Synthetic route of acceptor molecules c-PDI2, nc-PDI2, PDI4 

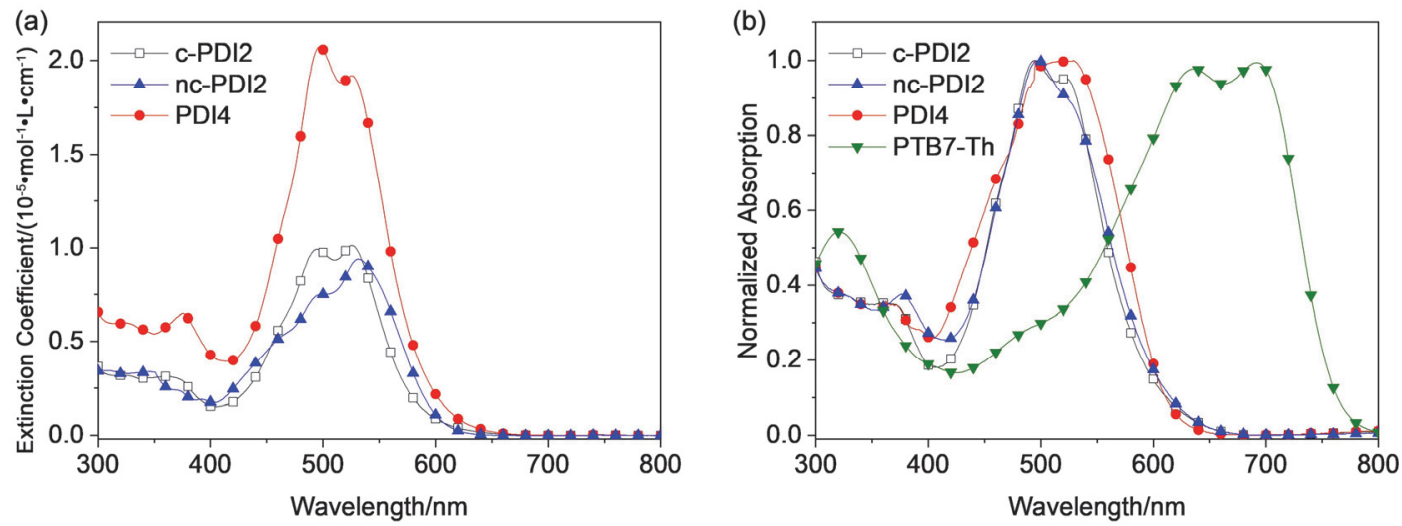

图 2 受体小分子在氯仿溶液 (a) 和薄膜 (b) 状态下的吸收光谱

Figure 2 Optical absorption curves of the acceptor molecules in chloroform solution (a) and thin films (b)

表 1 受体小分子的光物理和电化学性能参数

Table 1 Photophysical and electrochemical parameters of the acceptor molecules

\begin{tabular}{|c|c|c|c|c|c|c|c|}
\hline \multirow{2}{*}{ Acceptor } & \multicolumn{2}{|c|}{ Solution } & \multirow{2}{*}{$\begin{array}{c}\text { Film } \\
\lambda_{\max } / \mathrm{nm}\end{array}$} & \multirow{2}{*}{$E_{\mathrm{g}}^{\mathrm{opt}} / \mathrm{eV}$} & \multirow{2}{*}{$\mathrm{HOMO} / \mathrm{eV}$} & \multirow{2}{*}{$\mathrm{LUMO} / \mathrm{eV}$} & \multirow{2}{*}{$E_{\mathrm{g}}^{\mathrm{CV}} / \mathrm{eV}$} \\
\hline & $\lambda_{\max } / \mathrm{nm}$ & $\varepsilon /\left(\mathrm{L} \cdot \mathrm{mol}^{-1} \cdot \mathrm{cm}^{-1}\right)$ & & & & & \\
\hline c-PDI2 & 520 & $1.01 \times 10^{5}$ & 525 & 2.00 & -5.86 & -3.80 & 2.06 \\
\hline nc-PDI2 & 520 & $0.94 \times 10^{5}$ & 524 & 2.01 & -5.90 & -3.82 & 2.08 \\
\hline PDI4 & 500 & $2.10 \times 10^{5}$ & 528 & 2.01 & -5.99 & -3.90 & 2.09 \\
\hline
\end{tabular}

的光谱吸收范围较为相似, 主要集中在 $400 \sim 600 \mathrm{~nm}$.

与 c-PDI2 和 nc-PDI2 相比, PDI4 在这一区间具有更强 的光吸收能力. 通过计算得到 PDI4 的摩尔消光系数为 $2.1 \times 10^{5} \mathrm{~L} \cdot \mathrm{mol}^{-1} \cdot \mathrm{cm}^{-1}$, 超过了 c-PDI2 $\left(1.01 \times 10^{5}\right.$ $\left.\mathrm{L} \cdot \mathrm{mol}^{-1} \cdot \mathrm{cm}^{-1}\right)$ 和 nc-PDI2 $\left(0.94 \times 10^{5} \mathrm{~L} \cdot \mathrm{mol}^{-1} \cdot \mathrm{cm}^{-1}\right)$ 的 两倍. 其中的原因除了四臂型受体小分子中的 PDI 单元 数是双臂型受体小分子的二倍以外, 还有电子离域范围 增加等因素 ${ }^{[39]}$. 另外, 三个受体小分子还体现出了相对 不同的光谱吸收形状. c-PDI2 在 500 和 $530 \mathrm{~nm}$ 的两处吸 收峰值相当, 而 nc-PDI2 和 PDI4 分别在 500 和 $530 \mathrm{~nm}$ 左右具有出了相对更高的吸收峰. 由此可见 PDI4 的吸 收光谱不仅仅是两个双臂型受体小分子的简单加合. 三 个受体小分子薄膜的吸收光谱见图 $2 b$ 所示. 在薄膜状 态下, c-PDI2 的吸收光谱曲线几乎没有红移, 说明 c-PDI2 分子在薄膜中的聚集和电子在分子间离域非常 有限, 表明该分子的电子传输能力相对较低. nc-PDI2 分子在薄膜状态下的吸收光谱相对于溶液状态有大约 $10 \mathrm{~nm}$ 的红移, 表现出了较强的分子间聚集和较好的电 子在分子间离域. 在三个分子中, PDI4 薄膜的光谱吸收 红移最为明显, 超过了 $20 \mathrm{~nm}$, 这一结果表明 PDI4 具有 最好的电子在分子间离域, 同时表明 PDI4 具有更强的 电子传输能力. 通过受体小分子在薄膜状态下的起始吸 收波长计算得到 c-PDI2, nc-PDI2 和 PDI4 的光学带隙 $\left(E_{\mathrm{g}}{ }^{\mathrm{opt}}\right)$ 分别为 $2.00,2.01$ 和 $2.01 \mathrm{eV}$, 表明三个受体小分 子均为宽带隙受体材料. 图 $2 \mathrm{~b}$ 中也给出给体聚合物材 料 PTB7-Th 薄膜的吸收光谱作为对照.PTB7-Th 的光吸 收范围主要在 $550 \sim 750 \mathrm{~nm}$, 与三个受体小分子均能形
成了很好的光谱吸收互补, 所制备的活性层可以有效吸 收波长为 $400 \sim 750 \mathrm{~nm}$ 内的光子，几乎覆盖了整个可见 光范围.相对于使用给体单元中心核的菲二酰亚胺受体

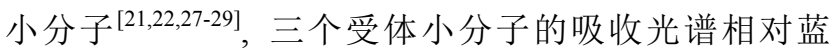
移 ${ }^{27,28]}$, 减少了和给体聚合物 PTB7-Th 的吸收范围的 重合, 从而改善了活性层对于太阳光吸收能力. 本工作 所发展的 PDI 受体小分子的光物理性能参数见表 1 所 示.

\section{3 受体小分子的电化学性能}

受体小分子的电化学性能通过循环伏安法 (CV) 来表征, 结果如图 3a 所示. c-PDI2、nc-PDI2 和 PDI4 的最高占有分子轨道与最低未占有分子轨道 $(\mathrm{HOMO} /$ LUMO) 能级分别为 $-5.86 /-3.80,-5.90 /-3.82$ 和 $-5.99 /-3.90 \mathrm{eV}$, 电化学带隙 $\left(E_{\mathrm{g}}{ }^{\mathrm{cV}}\right)$ 分别为 $2.06,2.08$ 和 $2.09 \mathrm{eV}$. 结果表明, c-PDI2, nc-PDI2 和 PDI4 均具有较 低的 LUMO 能级, 这是因为三个受体小分子均由缺电 子的 DPP 单元和 PDI 单元来构筑. 相对于使用给体单元 中心核的菲二酰亚胺小分子, 三个基于 DPP 中心核的 PDI 受体小分子具有相似的 LUMO 能级和更低的 HOMO 能级 ${ }^{[21,22,27-29]}$, 这也是造成了其吸收光谱相对蓝 移的原因. 如图 3b 所示, PTB7-Th 的 HOMO/LUMO 能 级为 $-5.20 /-3.56 \mathrm{eV}^{[40]}$, 与 PDI 受体小分子的能级也 能很好匹配. 本工作中所发展的 PDI 类受体小分子的电 化学性能参数见表 1 所示.

\section{4 受体小分子的分子模拟}

为了验证这三个受体小分子的扭曲分子结构, 我们 

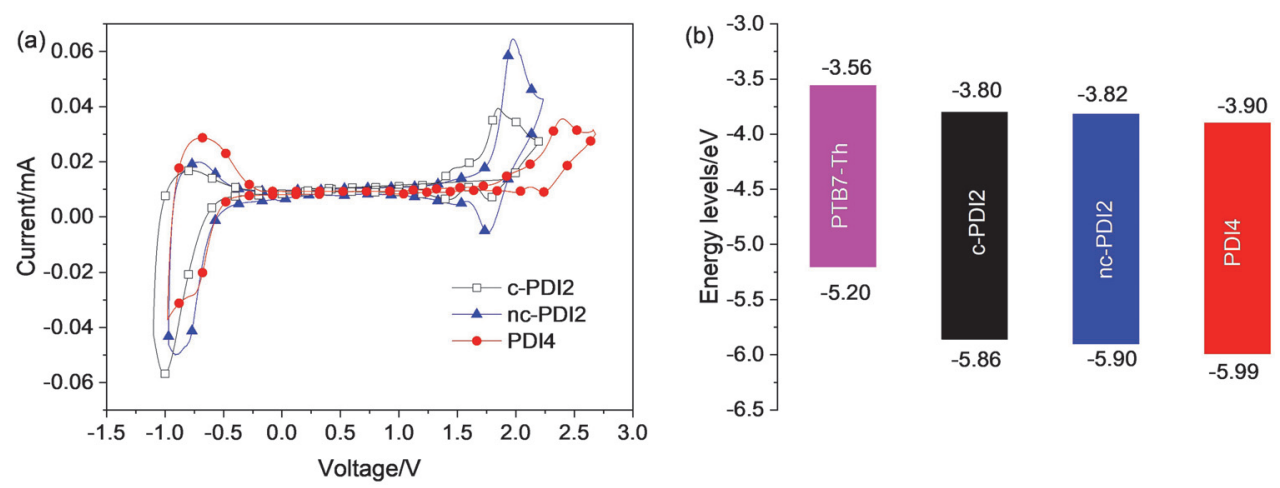

图 3 受体小分子的循环伏安曲线 (a) 和给受体材料的分子轨道能级分布 (b)

Figure 3 Cyclic voltammograms of the acceptor molecules (a) and energy levels of the acceptor molecules and the donor polymer (b)

使用密度泛函数方法 (DFT) 对受体小分子构型以及电 子云分布进行了模拟计算. 为了简化计算, 模型化合物 的长烷基链均用甲基替代, 因为这样不会显著影响分子 的几何构型和电子性质. 从侧视图 (图 S2) 可以看出, 各个 PDI 臂单元都处在不同的平面上, 这种空间构型可 以有效降低 PDI 分子自聚集 ${ }^{[21-24,26-29]}$. 其中, c-PDI2 与 nc-PDI2 的两个 PDI 单元之间呈现出错位平行构型, nc-PDI2 的两个 PDI 单元与苯环桥之间的二面角 $\left(35.0^{\circ} \sim 35.6^{\circ}\right)$ 明显小于 c-PDI2 的两个 PDI 单元与苯环 桥之间的二面角 $\left(54.5^{\circ} \sim 55.4^{\circ}\right)$. 这表明 nc-PDI2 的扭曲 程度较 c-PDI2 有所降低, 有助于其分子间堆积, 这与光 物理测试中得到的结论吻合. 与它们不同, PDI4 对位的 两个 PDI 单元在空间上是相互交叉而非相互平行. PDI4 分子中对应的大部分二面角都较 c-PDI2 和 nc-PDI2 有 所减小.从侧视图还可以看到, PDI4 呈现出独特的梭形 结构, 两端较平, 而中间隆起, 这有利于在获得较好分 子平面性的同时抑制分子的过度聚集. 从前线轨道分布 图可以看出, HOMO 轨道主要分布在中间核上, 而 LUMO 轨道则主要分布在电负性更大的 PDI 单元上. PDI4 的前线轨道具有更大的电子离域, 有望获得更高 的电子迁移率. 通过分子模拟计算得到的 c-PDI2, nc-PDI2 和 PDI4 的 HOMO/LUMO 能级分别为 -5.41 / $-3.47,-5.48 /-3.38$ 和 $-5.57 /-3.75 \mathrm{eV}$, 相应的带隙 分别为 $1.94,2.10$ 和 $1.82 \mathrm{eV}$, 这与电化学测试结果基本 一致.

\section{5 受体小分子的光伏性能}

我们使用三个受体小分子与窄带隙给体聚合物 PTB7-Th 共混作为活性层, 制作了结构为 ITO/ZnO/活 性层 $/ \mathrm{MoO}_{3} / \mathrm{Al}$ 的聚合物太阳能电池器件. 当活性层用 溶液旋涂法制备, 溶液配比中的给体、受体质量比为 $1: 1.5$ (材料总浓度为 $25 \mathrm{mg} / \mathrm{mL}$ ), 氯仿为溶剂, 溶剂添 加剂为 1,8 -二碘辛烷 (体积分数为 $0.85 \%$ ) 时, 器件获 得了最好的效率. 图 4a 给出了在这一优化条件下的聚 合物太阳能电池器件的 $J-V$ 曲线, 器件的光伏性能参数 总结在表 2 中. 在基于三个受体小分子的器件中, 双臂
型受体小分子 c-PDI2 器件 $\left(V_{\mathrm{oc}}=0.94 \mathrm{~V}\right)$ 和 nc-PDI2 器 件 $\left(V_{\mathrm{oc}}=0.93 \mathrm{~V}\right)$ 的 $V_{\mathrm{oc}}$ 明显高于四臂型受体小分子 PDI4 器件 $\left(V_{\mathrm{oc}}=0.85 \mathrm{~V}\right)$, 这是由于 PDI4 具有相对较低 的 LUMO 能级所导致. 但是四臂型 PDI4 器件则具有更 好的 $J_{\mathrm{sc}}$ 和 $\mathrm{FF}\left(J_{\mathrm{sc}}=15.26 \mathrm{~mA} \cdot \mathrm{cm}^{-2}, \mathrm{FF}=65.2\right)$, 明显高 于双臂型受体小分子 c-PDI2 器件 $\left(J_{\mathrm{sc}}=8.54 \mathrm{~mA} \cdot \mathrm{cm}^{-2}\right.$, $\mathrm{FF}=50.3)$ 和 nc-PDI2 器件 $\left(J_{\mathrm{sc}}=11.27 \mathrm{~mA} \cdot \mathrm{cm}^{-2}, \mathrm{FF}=\right.$ 65.15). 最终 PDI4 器件获得了最高的 PCE, 达到了 $8.45 \%$, 远高于了 c-PDI2 器件的 $4.04 \%$ 和 nc-PDI2 器件 的 $5.68 \%$.

如上所述, PDI4 具有更高器件性能的原因主要来 自于其更高的 $J_{\mathrm{sc}}$ 和 FF. 为了探究 $J_{\mathrm{sc}}$ 差异的机理, 我们 测量了三种器件的外量子效率 (EQE) 曲线, 如图 $4 \mathrm{~b}$ 所 示. 三个器件的 $\mathrm{EQE}$ 响应波长范围非常类似, 都主要集 中在 300 $800 \mathrm{~nm}$, 且曲线形状相似. c-PDI2, nc-PDI2 和 PDI4 器件的最大 EQE 响应值分别为 $43 \%, 50 \%$ 和 $75 \%$. 由此可见, 四臂型 PDI4 器件的 EQE 响应明显高 于双臂型受体小分子器件. PDI4 器件在 380 和 $500 \mathrm{~nm}$ 左右具有相对较高的 $\mathrm{EQE}$ 响应也与其较高光子吸收波 长对应，这表明 PDI4 较高的光子吸收能力是其导致其 器件具有较高 $J_{\mathrm{sc}}$ 的原因之一. 在波长大于 $600 \mathrm{~nm}$ 的给 体聚合物光吸收区间内, PDI4 器件也有着更高的 EQE 响应，这说明除了光吸收能力以外，还有其他原因导致 了 $J_{\mathrm{sc}}$ 的提升.

\section{6 活性层的电荷迁移率}

为了更加深入分析 PDI4 器件具有更高 $J_{\mathrm{sc}}$ 和 FF 的 原因, 我们运用空间电荷限制电流法 (SCLC) 对各器 件活性层的电荷迁移率进行了表征.测试结果如图 5 所 示, 根据对暗电流曲线拟合分析得到 c-PDI2, nc-PDI2 和 PDI4 与 PTB7-Th 共混薄膜的空穴/电子迁移率 $\left(\mu_{\mathrm{h}} / \mu_{\mathrm{e}}\right)$ 分别为 $1.94 \times 10^{-5} / 1.24 \times 10^{-5}, 1.09 \times 10^{-4} / 7.87 \times 10^{-5}$, $4.23 \times 10^{-4} / 3.11 \times 10^{-4} \mathrm{~m}^{2} \cdot \mathrm{V}^{-1} \cdot \mathrm{s}^{-1}$. 其空穴和电子迁移 率的比值分别为 $1.56,1.38$ 和 1.36. 显然, PTB7-Th:PDI4 薄膜具有最高的电荷迁移率和更加平衡的空穴和电子 传输能力. 更高和更平衡的电荷传输能力可以有效抑制 

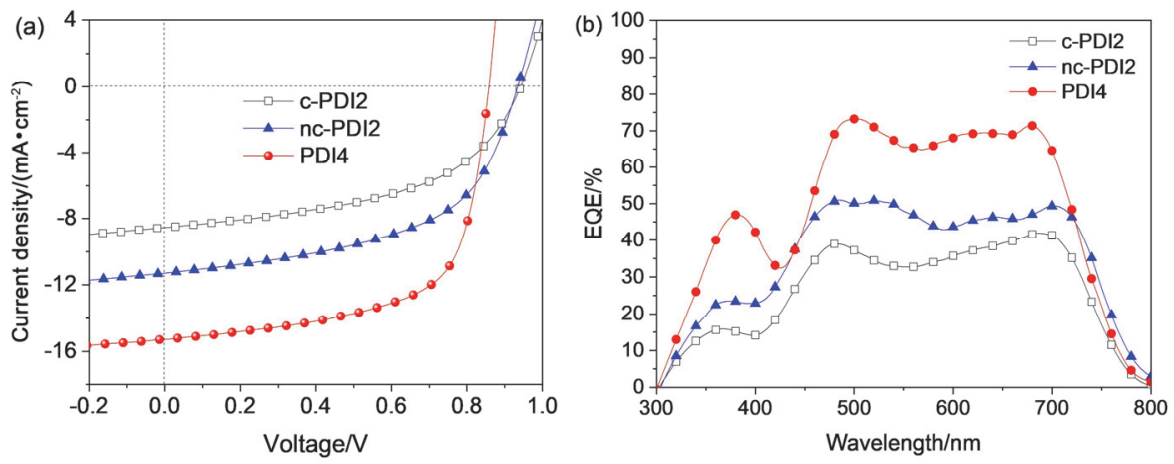

图 4 聚合物太阳能电池的 $J-V$ 曲线(a)和外量子效率曲线(b)

Figure $4 J-V$ curves (a) and EQE curves (b) of the polymer solar cells

表 2 聚合物太阳能电池的性能参数

Table 2 Photovoltaic parameters of the polymer solar cells

\begin{tabular}{cccccc}
\hline Acceptor & $V_{\mathrm{Oc}} / \mathrm{V}$ & $J_{\mathrm{sc}} / \mathrm{mA} \bullet \mathrm{cm}^{-2}$ & $J_{\mathrm{EQE}} /\left(\mathrm{mA} \bullet \mathrm{cm}^{-2}\right)$ & $\mathrm{FF} / \%$ & $\mathrm{PCE} / \%$ \\
\hline c-PDI2 & $0.94(0.93 \pm 0.01)$ & $8.54(8.38 \pm 0.14)$ & 8.50 & $50.3(48.2 \pm 1.6)$ & $4.04(3.77 \pm 0.17)$ \\
nc-PDI2 & $0.93(0.92 \pm 0.01)$ & $11.27(10.87 \pm 0.35)$ & 11.20 & $54.2(51.9 \pm 1.7)$ & $5.68(5.20 \pm 0.29)$ \\
PDI4 & $0.85(8.84 \pm 0.02)$ & $15.26(15.05 \pm 0.20)$ & 15.24 & $65.2(62.9 \pm 2.0)$ & $8.45(7.93 \pm 0.32)$ \\
\hline
\end{tabular}
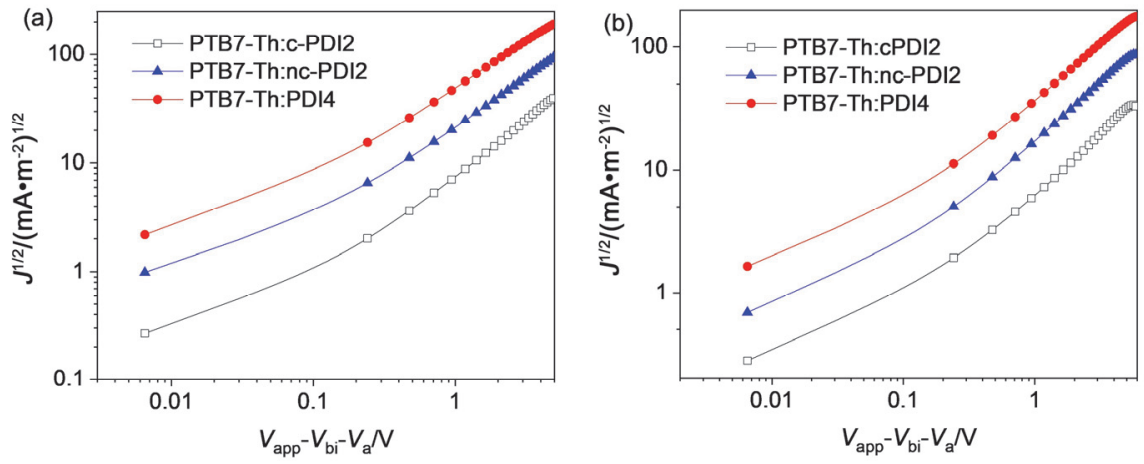

图 5 单空穴器件(a) 和单电子器件(b) 的电流 $\left(J^{1 / 2}\right)$-电压 $(V)$ 特性曲线

Figure $5 J^{1 / 2}-V$ curves of the hole-only (a) and the electron-only (b)devices

载流子复合, 从而显著提高器件的 $J_{\mathrm{sc}}$ 和 FF. 如前所述, PDI4 具有更强的电子传输能力, 这与其分子内和分子 间电子云离域范围更广有关. 比较两个双臂型受体小分 子, PTB7-Th:nc-PDI2 薄膜的电荷传输能力明显高于 PTB7-Th:c-PDI2, 而且电荷传输更加平衡, 这和 nc-PDI2 光物理性能和分子模拟中体现出的更好分子平 面性和更强的分子间固态堆积相关, 这也是造成两个双 臂型分子器件性能差异的主要原因.

\section{7 活性层的形貌表征}

我们利用 $X$ 射线衍射(XRD) 测试研究了基于这三 个受体小分子的共混薄膜的结晶情况, 结果如图 6 所示. 三个共混薄膜都出现了两个衍射峰, 其中 $2 \theta$ 值在 $4.3^{\circ}$ 附近的较尖锐的衍射峰是给体聚合物 PTB7-Th 的层间 堆积(100) 衍射峰, 计算得到其相应的层间堆积距离为

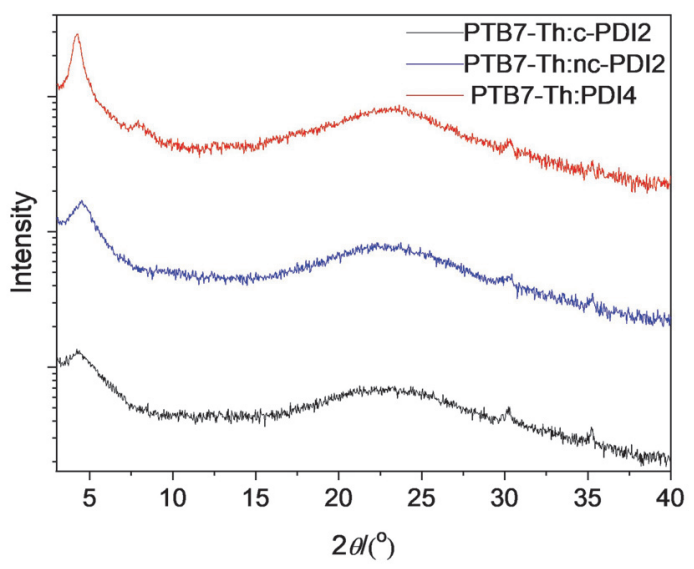

图 6 共混薄膜的 X 衍射曲线

Figure 6 X-ray diffraction patterns of the blend films 
$2.05 \mathrm{~nm}$. 而 $2 \theta$ 值约等于 $23^{\circ}$ 的相对宽阔的衍射峰是 PTB7-Th 的 $\pi-\pi$ 堆积 (010) 衍射峰, 计算得到其相应的 层间堆积距离为 $3.9 \AA$. PTB7-Th 在与 c-PDI2、nc-PDI2 和 PDI4 共混时, 其共混膜的衍射强度逐渐增强. 在含 有 PDI4 的共混薄膜中, PTB7-Th 的 (200) 峰在 $2 \theta$ 值为 $8^{\circ}$ 左右可见，这进一步说明了在这一薄膜中 PTB7-Th 的 结晶度更高. 更高的聚合物给体结晶度可以提高活性层 薄膜的空穴迁移率, 进而进一步提高器件的 $J_{\mathrm{sc}}$ 和 FF. 除了聚合物的衍射峰以外, 在三个薄膜中都没有检测到 其他的衍射峰. 由此我们可以判定, 三个受体小分子都 没有在薄膜中结晶, 处于无定形状态, PDI 单元的自聚 集趋势在三个受体小分子中都得到了很好的抑制.

为了进一步表征活性层薄膜的纳米相分离情况, 我 们用原子力显微镜 (AFM) 对器件的活性层薄膜形貌 进行了测试, 结果如图 7 中的高度图和相图所示. PTB7-Th 与 c-PDI2, nc-PDI2 和 PDI4 共混的薄膜都展 示了均匀和平滑的表面结构, 其均方根粗䊁度(RMS) 分别为 $3.20,2.51$ 和 $2.99 \mathrm{~nm}$. 三个薄膜中均出现了数十 纳米尺度的斑块状形貌, 但并未出现 PDI 薄膜中常见的 微米尺度的块状晶体, 这说明了 PDI 的大尺度结晶和聚 集得到了有效的抑制. 三个共混薄膜的表面形貌也与 $\mathrm{X}$ 射线衍射测试中聚合物结晶为主的纳米相分离结构相 吻合.

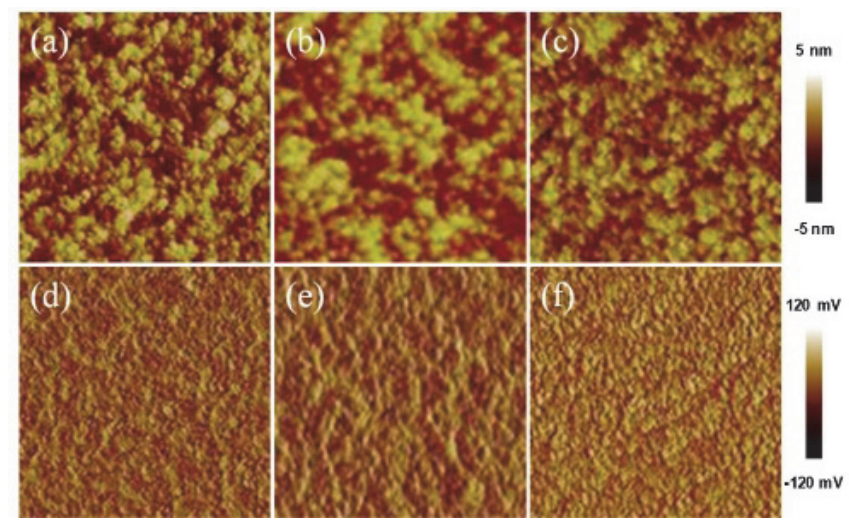

图 7 基于 c-PDI2 $(\mathrm{a}, \mathrm{d}), \mathbf{n c - P D I 2}(\mathrm{b}, \mathrm{e})$ 和 PDI4 $(\mathrm{c}, \mathrm{f})$ 的共混薄膜的原 子力显微镜高度图 $(\mathrm{a}, \mathrm{b}, \mathrm{c})$ 和相图 $(\mathrm{d}, \mathrm{e}, \mathrm{f})$, 图片宽度为 $5 \mu \mathrm{m}$ Figure 7 AFM height $(\mathrm{a}, \mathrm{b}, \mathrm{c})$ and phase $(\mathrm{d}, \mathrm{e}, \mathrm{f})$ images of the blend films based on c-PDI2 (a,d), nc-PDI2 (b,e) and PDI4 (c,f), the image widths are $5 \mu \mathrm{m}$

\section{3 结论}

在本文中, 我们设计合成了三个基于吡咯并吡咯二 酮 (DPP) 为中心核的荘二酰亚胺 (PDI) 类受体小分子 c-PDI2, nc-PDI2 和 PDI4, 系统研究了其结构和性能之 间的关系. 其中 c-PDI2 和 nc-PDI2 分别为将两个 PDI 臂单元键接在 DPP 骨架的碳取代位和氮取代位上, 而 PDI4 则是将四个 PDI 臂单元键接在 DPP 骨架的四个不 同取代位上. 三个受体小分子都具有扭曲的空间构型, 有效抑制了受体小分子的自聚集趋势. 相对于文献报道
的基于给体单元中心核的菲二酰亚胺受体小分子，本工 作引入 DPP 受体中心核降低了 PDI 受体分子的 HOMO 能级, 增大了其能带隙, 导致光谱吸收范围相对蓝移, 提高了与给体聚合物 PTB7-Th 吸收光谱的匹配度. 通 过对两个双臂型受体小分子的研究，氮位取代的 nc-PDI2 表现出了更高的电子传输性能，从而获得了相 对更好的器件效率.相对于双臂型受体小分子, 四臂型 PDI4 则体现出很更好的光谱吸收能力和电子传输性能, 从而获得了 $8.45 \%$ 的最高能量转换效率, 分别是 $\mathbf{c}-\mathbf{P D I} 2$ 器件的 2 倍和 nc-PDI2 器件的 1.5 倍.

\section{4 实验部分}

\section{1 受体小分子合成}

受体小分子 c-PDI2 的合成: 氮气保护下, 将碳酸钾 $(1.38 \mathrm{~g}, 10 \mathrm{mmol})$ 的水 $(5 \mathrm{~mL})$ 溶液加入含有化合物 PDI-Bpin $(0.50 \mathrm{~g}, 0.60 \mathrm{mmol})$ 和 c-DPP-Br2 $(0.20 \mathrm{~g}, 0.30$ $\mathrm{mmol})$ 的甲苯 $(10 \mathrm{~mL})$ 溶液中, 随后加入 $2.5 \mathrm{~mL}$ 的无 水乙醇和 $\mathrm{Pd}\left(\mathrm{PPh}_{3}\right)_{4}(0.04 \mathrm{~mol})$, 在 $120{ }^{\circ} \mathrm{C}$ 避光反应 $18 \mathrm{~h}$. 冷却后, 用冰水淬灭反应, 用二氯甲烷萃取反应液, 分 离有机相并用无水硫酸镁干燥, 过滤, 旋蒸除去溶剂.粗 产物经过石油醚-二氯甲烷 $\left(V_{\text {石油瞵 }}: V\right.$ 二氯甲㟋 $\left.=1: 3\right)$ 为 流动相的硅胶柱层析分离提纯得到暗红色固体产物 c-PDI2 (0.22 g, 产率 40\%) . ${ }^{1} \mathrm{H}$ NMR (400 $\mathrm{MHz}, \mathrm{CDCl}_{3}$ ) $\delta: 8.80 \sim 8.58(\mathrm{~m}, 10 \mathrm{H}), 8.11(\mathrm{~d}, J=38.5 \mathrm{~Hz}, 6 \mathrm{H}), 7.96(\mathrm{~d}$, $J=8.3 \mathrm{~Hz}, 2 \mathrm{H}), 7.73(\mathrm{~d}, J=7.9 \mathrm{~Hz}, 4 \mathrm{H}), 5.32 \sim 5.07(\mathrm{~m}$, $4 \mathrm{H}), 3.90(\mathrm{~d}, J=7.4 \mathrm{~Hz}, 4 \mathrm{H}), 2.35 \sim 2.13(\mathrm{~m}, 10 \mathrm{H}), 1.96 \sim$ $1.75(\mathrm{~m}, 8 \mathrm{H}), 1.39 \sim 1.15(\mathrm{~m}, 64 \mathrm{H}), 0.97 \sim 0.75(\mathrm{~m}, 36 \mathrm{H})$; ${ }^{13} \mathrm{C}$ NMR $\left(100 \mathrm{MHz}, \mathrm{CDCl}_{3}\right) \delta: 166.31,166.12,155.62$, $154.37,153.59,145.98,140.81,138.52,138.37,134.26$, $132.47,131.45,128.10,127.49,126.18,124.56,122.81$, 119.07, 113.13, 112.08, 56.35, 56.06, 40.80, 36.79, 26.80, 22.99 , 14.24, 13.95; Anal. calcd for $\mathrm{C}_{126} \mathrm{H}_{152} \mathrm{~N}_{6} \mathrm{O}_{10}$ : C 79.21, H 8.02, N 4.40; found: C 79.30, H 8.08, N 4.90; MS (MALDI-TOF) calcd for $\mathrm{C}_{126} \mathrm{H}_{152} \mathrm{~N}_{6} \mathrm{O}_{10}$ 1909.157, found 1909.230.

受体小分子 nc-PDI2 的合成: 氮气保护下，将碳酸 钾 $(1.38 \mathrm{~g}, 10 \mathrm{mmol})$ 的水 $(5 \mathrm{~mL})$ 溶液加入含有化合物 PDI-Bpin (0.74 g, $0.90 \mathrm{mmol})$ 和 nc-DPP-Br2 (0.20 g, $0.27 \mathrm{mmol})$ 的甲苯 $(10 \mathrm{~mL})$ 溶液中, 并加入 $2.5 \mathrm{~mL}$ 的 无水乙醇和 $\mathrm{Pd}\left(\mathrm{PPh}_{3}\right)_{4}(0.04 \mathrm{~mol})$, 在 $120{ }^{\circ} \mathrm{C}$ 避光反应 18 h. 冷却后, 用冰水淬灭反应, 用二氯甲烷萃取反应液, 分离有机相并用无水硫酸镁干燥, 过滤, 旋蒸除去溶剂. 粗产物经过石油醚-二氯甲烷 $\left(V_{\text {石油瞵 }}: V_{\text {二氯甲烷 }}=3: 1\right)$ 为流动相的硅胶柱层析分离提纯得到暗红色固体产物 nc-PDI2 (0.14 g, 产率 65\%) ${ }^{1} \mathrm{H}$ NMR (400 $\left.\mathrm{MHz}, \mathrm{CDCl}_{3}\right)$ $\delta: 8.78 \sim 8.58(\mathrm{~m}, 8 \mathrm{H}), 8.27 \sim 8.06(\mathrm{~m}, 2 \mathrm{H}), 7.95(\mathrm{t}, J=8.6$ $\mathrm{Hz}, 2 \mathrm{H}), 7.17 \sim 7.08(\mathrm{~d}, J=8.1 \mathrm{~Hz}, 4 \mathrm{H}), 7.08$ (d, $J=8.1$ $\mathrm{Hz}, 2 \mathrm{H}), 7.26 \sim 6.94(\mathrm{~m}, 4 \mathrm{H}), 6.94 \sim 6.68(\mathrm{~m}, 4 \mathrm{H}), 6.23 \sim$ 
$6.00(\mathrm{~m}, 4 \mathrm{H}), 5.25 \sim 5.09(\mathrm{~m}, 4 \mathrm{H}), 2.34 \sim 2.12(\mathrm{~m}, 8 \mathrm{H})$, $1.93 \sim 1.65(\mathrm{~m}, 12 \mathrm{H}), 1.41 \sim 1.17(\mathrm{~m}, 72 \mathrm{H}), 0.91 \sim 0.79$ $(\mathrm{m}, 30 \mathrm{H}) . .{ }^{13} \mathrm{C}$ NMR $\left(100 \mathrm{MHz}, \mathrm{CDCl}_{3}\right) \delta: 166.51,165.12$, $155.62,154.37,153.59,144.98,140.81,139.52$, 138.37, $134.26,132.47,131.45,128.10,127.49,127.18,124.56$, 122.81, 118.07, 113.13, 112.08, 56.35, 56.06, 40.80, 36.79, 26.80, 22.99, 14.24, 13.95. Anal. calcd for $\mathrm{C}_{138} \mathrm{H}_{160} \mathrm{~N}_{6} \mathrm{O}_{10}$ : C 80.35, H 7.82, N 4.07; found: C 80.65, H 8.02, N 4.37; MS (MALDI-TOF) calcd for $\mathrm{C}_{138} \mathrm{H}_{160} \mathrm{~N}_{6} \mathrm{O}_{10}$ 2061.220, found 2061.220.

受体小分子 PDI4 的合成: 氮气保护下, 将碳酸钾 $(1.38 \mathrm{~g}, 10 \mathrm{mmol})$ 的水 $(5 \mathrm{~mL})$ 溶液加入化合物 PDI-Bpin $(0.74 \mathrm{~g}, 0.90 \mathrm{mmol})$ 和 DPP-Br4 (0.10 g, 0.13 $\mathrm{mmol})$ 的甲苯 $(10 \mathrm{~mL})$ 溶液中, 并加入 $2.5 \mathrm{~mL}$ 的无水 乙醇和 $\mathrm{Pd}\left(\mathrm{PPh}_{3}\right)_{4}(0.04 \mathrm{~mol})$, 在 $120{ }^{\circ} \mathrm{C}$ 避光反应 $24 \mathrm{~h}$. 冷却后, 用冰水淬灭反应, 用二氯甲烷萃取反应液, 分 离有机相并用无水硫酸镁干燥, 过滤, 旋蒸除去溶剂. 粗 产物经过石油醚-二氯甲烷 $(V$ 石油瞵 $: V$ 二氯甲烷 $=5: 1)$ 为 流动相的硅胶柱层析分离提纯得到暗红色固体产物 PDI4(0.23 g, 产率 54\%) ${ }^{1} \mathrm{H}$ NMR (400 MHz, $\left.\mathrm{CDCl}_{3}\right) \delta$ : $8.78 \sim 8.50(\mathrm{~m}, 20 \mathrm{H}), 8.21 \sim 8.12(\mathrm{~m}, 8 \mathrm{H}), 7.95(\mathrm{t}, J=8.6$ $\mathrm{Hz}, 4 \mathrm{H}), 7.73$ (d, $J=8.1 \mathrm{~Hz}, 4 \mathrm{H}), 7.66(\mathrm{~d}, J=8.1 \mathrm{~Hz}, 4 \mathrm{H})$, $7.58(\mathrm{~d}, J=8.3 \mathrm{~Hz}, 4 \mathrm{H}), 5.14 \sim 5.18(\mathrm{~m}, 8 \mathrm{H}), 2.35 \sim 2.05$ $(\mathrm{m}, 16 \mathrm{H}), 1.80 \sim 1.82(\mathrm{~m}, 16 \mathrm{H}), 1.36 \sim 1.14(\mathrm{~m}, 96 \mathrm{H})$, $0.85 \sim 0.72(\mathrm{~m}, 48 \mathrm{H}) .{ }^{13} \mathrm{C} \mathrm{NMR}\left(100 \mathrm{MHz}, \mathrm{CDCl}_{3}\right) \delta$ : $164.91,159,76,159.10,155.62,154.37,153.59,145.98$, $140.81,138.52,138.37,134.26,132.47,131.45,128.10$, $127.49,126.18,124.56,122.81,119.07,113.13,112.08$, 56.35, 56.06, 40.80, 36.79, 26.80, 22.99, 14.24, 13.95. Anal. calcd for $\mathrm{C}_{214} \mathrm{H}_{236} \mathrm{~N}_{10} \mathrm{O}_{18}$ : C 79.42, H 7.35, N 4.33; found: $\mathrm{C} 79.52$, $\mathrm{H} 7.65, \mathrm{~N}$ 4.63; MS (MALDI-TOF) calcd for $\mathrm{C}_{214} \mathrm{H}_{236} \mathrm{~N}_{10} \mathrm{O}_{18} 3233.786$, found 3233.806.

\section{2 太阳能电池器件制备与测试}

ITO 导电玻璃依次用去离子水、丙酮、异丙醇超声 清洗后放入真空干燥箱干燥. 干燥后的 ITO 用臭氧处理 $15 \mathrm{~min}$. 随后在 ITO 表面旋涂一层厚度约 $30 \mathrm{~nm}$ 的氧化锌 薄膜. 随后在干燥后的氧化锌表面旋涂含有 PTB7-Th 和 受体小分子的活性层. 将器件放入高真空蒸镀仓, 在活 性层表面蒸镀三氧化钼空穴传输层和铝电极, 厚度分别 为 $10 \mathrm{~nm}$ 和 $30 \mathrm{~nm}$. 器件的有效面积通过掩膜板调控为 $0.04 \mathrm{~cm}^{2}$. 器件的 $I-V$ 曲线在 1 个模拟太阳光下(Japan, SAN-EI, XES-70S1), 通过 Keithley 2400 采集. 太阳光模 拟器采用标准的硅电池标定为 $100 \mathrm{~mW} \cdot \mathrm{cm}^{-2}$. 器件的 $\mathrm{EQE}$ 曲线在氙气光源单色光条件下由 Newport $\mathrm{QE}$ 测试 系统测得. 所有测试过程都在氩气填充的手套箱中完 成.

\section{3 电荷迁移率测试}

电荷迁移率的测试方法采用 SCLC 方法. 测定空穴
迁移率的器件结构为 ITO/PEDOT:PSS/活性层/ $\mathrm{MoO}_{3} / \mathrm{Al}$, 测定电子迁移率的器件结构为 $\mathrm{Al} /$ 活性层/Al. 器件制备 过程中. PEDOT:PSS 层和活性层均由溶液旋涂法制备, 三氧化钼和铝电极层由真空蒸镀制备. 电荷迁移率由 Keithley 2400 测量所得到的 $I-V$ 曲线拟合计算得到.

\section{References}

[1] Inganäs, O. Adv. Mater. 2018, 30, 1800388.

[2] Cheng, P.; Li, G.; Zhan, X. W.; Yang, Y. Nat. Photonics 2018, 12, 131.

[3] Hou, J. H.; Inganäs, O.; Friend, R. H.; Gao, F. Nat. Mater. 2018, 17, 119.

[4] Wadsworth, A.; Moser, M.; Marks, A.; Little, M. S. S.; Gasparini, N.; Brabec, C. J. J.; Baran, D.; McCulloch, I. Chem. Soc. Rev. 2019, 48, 1596.

[5] Yan, C. Q.; Barlow, S.; Wang, Z. H.; Yan, H.; Jen, A. K. Y.; Marder, S. R.; Zhan, X. W. Nat. Rev. Mater. 2018, 3, 18003.

[6] Xu, X. P.; Feng, K.; Lee, Y. W.; Woo, H. Y.; Zhang, G. J.; Peng, Q. Adv. Funct. Mater. 2020, 30, 1907570.

[7] Yan, T. T.; Song, W.; Huang, J. M.; Peng, R. X.; Huang, L. K.; Ge, Z. Y. Adv. Mater. 2019, 31, 1902210.

[8] Yu, R. N.; Yao, H. F.; Cui, Y.; Hong, L.; He, C.; Hou, J. H. Adv. Mater. 2019, 31, 1902302.

[9] Xu, X. P.; Feng, K.; Bi, Z. Z.; Ma, W.; Zhang, G. J.; Peng Q. Adv Mater. 2019, 31, 1901872.

[10] Sun, H. L.; Liu, T.; Yu, J. W.; Lau, T. K.; Zhang, G. Y.; Zhang, Y. J.; Su, M. Y.; Tang, Y. M.; Ma, R. J.; Liu, B.; Liang, J. E.; Feng, K.; Lu, X. H.; Guo, X. G.; Gao, F.; Yan, H. Energy Environ. Sci. 2019, 12, 3328.

[11] Cui, Y.; Yao, H. F.; Zhang, J. Q.; Zhang, T.; Wang, Y. M.; Hong, L.; Xian, K. H.; Xu, B. W.; Zhang, S. Q.; Peng, J.; Wei, Z. X.; Gao, F.; Hou, J. H. Nat. Commun. 2019, 10, 2515.

[12] Duan, Y. W.; Xu, X. P.; Yan, H.; Wu, W. L.; Li, Z. J.; Peng, Q. Adv. Mater. 2017, 29, 1605115

[13] Genene, Z.; Mammo, W.; Wang, E. G.; Andersson, M. R. Adv. Mater. 2019, 31, 1807275.

[14] Zhang, G. Y.; Zhao, J. B.; Chow, P. C. Y.; Jiang, K.; Zhang, J. Q.; Zhu, Z. L.; Huang, F.; Yan, H. Chem. Rev. 2018, 118, 3447.

[15] Zhang, J. Q.; Tan, H. S.; Guo, X. G.; Facchetti, A.; Yan, H. Nat. Energy 2018, 3, 720 .

[16] Hartnett, P. E.; Timalsina, A.; Matte, H. S. S. R.; Zhou, N. J.; Guo, X. G.; Zhao, W.; Facchetti, A.; Chang, R. P. H.; Hersam, M. C.; Wasielewski, M. R.; Marks, T. J. J. Am. Chem. Soc. 2014, 136, 16345.

[17] Zhong, Y.; Trinh, M. T.; Chen, R. S.; Wang, W.; Khlyabich, P. P.; Kumar, B.; Xu, Q. Z.; Nam, C. Y.; Sfeir, M. Y.; Black, C.; Steigerwald, M. L.; Loo, Y. L.; Xiao, S. X.; Ng, F.; Zhu, X. Y.; Nuckolls, C. J. Am. Chem. Soc. 2014, 136, 15215.

[18] Eastham, N. D.; Dudnik, A. S.; Aldrich, T. J.; Manley, E. F.; Fauvell, T. J.; Hartnett, P. E.; Wasielewski, M. R.; Chen, L. X.; Melkonyan, F. S.; Facchetti, A.; Chang, R. P. H.; Marks, T. J. Chem. Mater. 2017, $29,4432$.

[19] Liang, N. N.; Sun, K.; Zheng, Z.; Yao, H. F.; Gao, G. P.; Meng, X. Y.; Wang, Z. H.; Ma, W.; Hou, J. H. Adv. Energy Mater. 2016, 6, 1600060 .

[20] Ye, L.; Sun, K.; Jiang, W.; Zhang, S. Q.; Zhao, W. C.; Yao, H. F.; Wang, Z. H.; Hou, J. H. ACS Appl. Mater. Interfaces 2015, 7, 9274.

[21] Yan, Q. F.; Zhou, Y.; Zheng, Y. Q.; Pei, J.; Zhao, D. H. Chem. Sci. 2013, 4, 4389.

[22] Wu, J. Z.; Li, X. C.; Liu, X. D.; Wu, S. H.; Lai, W. Y.; Zheng, Y. H. J. Mater. Chem. C 2018, 6, 13171 .

[23] Meng, D.; Sun, D.; Zhong, C. M.; Liu, T.; Fan, B. B.; Huo, L. J.; Li, Y.; Jiang, W.; Choi, H. S.; Kim, T.; Kim, J. Y.; Sun, Y. M.; Wang, Z. H.; Heeger, A. J. J. Am. Chem. Soc. 2016, 138, 375.

[24] Kim, H. S.; Park, H. J.; Lee, S. K.; Shin, W. S.; Song, C. E.; Hwang, D. H. Org. Electron. 2019, 71, 238.

[25] Kushwaha, K.; Yu, L. Y.; Stranius, K.; Singh, S. K.; Hultmark, S.; Iqbal, M. N.; Eriksson, L.; Johnston, E.; Erhart, P.; Müller, C.; Börjesson, K. Adv. Sci. 2019, 6, 1801650.

[26] Lin, Y. Z.; Wang, Y. F.; Wang, J. Y.; Hou, J. H.; Li, Y. F.; Zhu, D. B.; Zhan, X. W. Adv. Mater. 2014, 26, 5137.

[27] Bian, G. F.; Zhao, F.; Lau, T. K.; Sheng, C. Q.; Lu, X. H.; Du, H.; 
Zhang, C.; Qu, Z. R.; Chen, H. Z.; Wan, J. H. J. Mater. Chem. C 2019, 7, 8092.

[28] Tang, F.; Wu, K. L.; Zhou, Z. J.; Wang, G.; Zhao, B.; Tan, S. T. ACS Appl. Energy Mater. 2019, 2, 3918.

[29] Ding, K.; Wang, Y.; Shan, T.; Xu, J. Q.; Bao, Q. Y.; Liu, F.; Zhong, H. L. Org. Electron. 2020, 78, 105569.

[30] Wang, K. K.; Xia, P.; Wang K. W.; You, X. X.; Wu, M. L.; Huang, H. X.; Wu, D.; Xia, J. L. ACS Appl. Mater. Interfaces 2020, 12, 9528.

[31] Zhang, G. J.; Xu, X. P.; Lee, Y. W.; Woo, H. Y.; Li, Y.; Peng, Q. $A d v$. Funct. Mater. 2019, 29, 1902079.

[32] Liu, X.; Liu, T.; Duan, C. H.; Wang, J. Y.; Pang, S. T.; Xiong, W. T.; Sun, Y. M.; Huang, F.; Cao, Y. J. Mater. Chem. A 2017, 5, 1713.

[33] Song, K. C.; Singh, R.; Lee, J.; Sin, D. H.; Lee, H.; Cho, K. J. Ma- ter. Chem. C 2016, 4, 10610.

[34] Li, Y. N.; Sonar, P.; Murphy, L.; Hong, W. Energy Environ. Sci. 2013, 6, 1684.

[35] Qu, S. Y.; Tian, H. Chem. Commun. 2012, 48, 3039.

[36] Chen, Z. Y.; Lee, M. J.; Ashraf, R. S.; Gu, Y.; Albert-Seifried, S.; Nielsen, M. M.; Schroeder, B.; Anthopoulos, T. D.; Heeney, M.; McCulloch, I.; Sirringhaus, H. Adv. Mater. 2012, 24, 647.

[37] Lin, Y. Z.; Li, Y. F.; Zhan, X. W. Adv. Energy Mater. 2013, 3, 724.

[38] Liu, S. Y.; Liu, W. Q.; Yuan, C. X.; Zhong, A. G.; Han, D. M.; Wang, B.; Shah, M. N.; Shi, M. M.; Chen, H. Z. Dye. Pigment. 2016, 134, 139.

[39] Jana, B.; Ghosh, A.; Patra, A. J. Phys. Chem. Lett. 2017, 8, 4608.

[40] Li, Y.; Gong, Y. F.; Che, Y. J.; Xu, X. P.; Yu, L. Y.; Peng, Q. Front. Chem. 2020, 8,1 .

(Yang, X.) 